\title{
The Importance of External Support in the Adoption of Open Source Server Software
}

\author{
Kris Ven and Jan Verelst \\ University of Antwerp, Prinsstraat 13, 2000 Antwerp, Belgium \\ \{kris.ven, jan.verelst\}@ua.ac.be
}

\begin{abstract}
IT managers seem to be hesitant to adopt OSS in the absence of professional support. Previous qualitative studies have indeed suggested that the availability of external support is important for the adoption of OSS. Therefore, we feel it is interesting to gain more insight into the role of external support in the adoption process. To this end, we performed a web survey involving 95 Belgian organizations. Our data suggests a balanced picture. As expected, our results show that the majority of organizations in our sample rely on commercial support such as vendor or third party support. Even organizations that have deployed OSS to a large extent-and that are therefore likely to have some experience and familiarity with OSS - rely on commercial support. Nevertheless, a considerable proportion of organizations indicated not to rely on commercial support, which suggests that internal expertise can be sufficient for successful adoption. Finally, and most surprisingly, we have found that the OSS community is used by a large proportion of organizations. This indicates that the OSS community is a valuable source of external support for organizations. Nevertheless, it appears that it is primarily used by organizations with a rather strong background in IT.
\end{abstract}

Keywords: Open source software, adoption, assimilation, knowledge, external support, OSS community.

\section{Introduction}

Several studies have suggested that the availability of external support is an important concern in the adoption of OSS [3|6 8/10 14]. Morgan and Finnegan have found that a lack of external support may be an important barrier to the adoption of OSS, especially for large organizations [10]. Similarly, Li et al. have found that the availability of external support did have an influence on the intention to adopt OSS [8]. In addition, it seems that the type of support chosen also differs from organization to organization [10]. The ability to rely on external support has been found to provide some reassurance to organizations [6]. These findings suggest that IT managers are very reluctant to adopt OSS without any external support, fearing that they cannot rely on the support that is offered by the OSS community or on the internal resources of the organization.

The results of our previous qualitative research were consistent with these observations [14]. Most organizations preferred being able to rely on at least some form of commercial support (e.g., a support contract or the services of a service provider) and indicated that a lack of commercial support may limit the adoption of OSS.

(C) IFIP International Federation for Information Processing 2009 
In a subsequent quantitative study, our aim was to identify the factors that influence the assimilation of open source server software (OSSS) [15]. Assimilation can be described as "an organizational process that (1) is set in motion when individual organization members first hear of an innovation's development, (2) can lead to the acquisition of the innovation, and (3) sometimes comes to fruition in the innovation's full acceptance, utilization, and institutionalization." [9]. Assimilation is therefore a broader term than adoption, which only refers to the decision on whether to adopt an innovation or not. The term OSSS was used in our study to refer to OSS that is primarily used on servers.

Interestingly, our quantitative study showed that although the availability of external support was important and decreases switching costs, it did not have a direct impact on the assimilation stage reached by organizations [15]. This suggests that the availability of external support has a similar impact on all assimilation stages. However, there are several indications from previous qualitative studies that external support for OSS is important. Therefore, it is interesting to further investigate the role of external support in the adoption of OSS. To this end, a follow-up survey among Belgian organizations was undertaken. The aim of this study was to gain insight into the way in which organizations rely on external support. Although it could be expected that external support is crucial for the adoption of OSS, our data suggests a somewhat more balanced view of external support.

The rest of the paper is structured as follows. We start with a brief description of the methodology that was used in this study. Subsequently, we provide the results of the quantitative analysis of the data obtained through the survey. This is followed by a discussion of our findings. Finally, some conclusions are offered.

\section{Methodology}

In order to study the role of external support in the assimilation of OSS, we performed a quantitative study. A self-administered web survey was used to collect the data for our study. The survey instrument was subjected to a qualitative pretest involving 5 experts to identify any issues in the survey design including, but not limited to, understanding and answering the questions in the survey. No major issues were discovered during this pretest, but some minor adjustments were made in the formulation of some questions.

Similar to our previous study [15], this survey was focused on the use of OSSS. For the purpose of this survey, the term OSSS referred to a limited list of 7 OSS products consisting of Linux, BSD, Apache, Bind, Sendmail, Postfix and Samba. Respondents were instructed on each page of the survey that the term OSSS referred to this specific list of OSS products.

The subjects for our study consisted of those respondents to our previous study [15] that indicated to be willing to participate in a follow-up survey. Our sample therefore consisted of Belgian organizations that had servers installed. Organizations from different sectors and sizes were represented in our sample. The target person in each organization was the IT decision maker, commonly the CIO or IT manager. In order to minimize any biases due to time delays between observations, the invitation to participate in the follow-up survey was sent one week after the respondent completed the first survey. The 
survey was administered between July and October 2007. Out of 332 respondents who responded to the first survey, $153(46.1 \%)$ were willing to participate in this follow-up survey. A total of 111 replies were received, which corresponds to a response rate of $72.5 \%$. This exceptionally high response rate can be attributed to a number of factors. First, each respondent was personally contacted by telephone to ask for his or her participation in the first survey. We observed that this had a very favourable influence on the tendency to cooperate. Second, we asked respondents if they wanted to participate in the follow-up survey, providing them with the possibility to decline. Those that agreed can be assumed to have very strong intentions to complete the survey. Finally, a number of reminders were sent if respondents did not complete the survey within two weeks.

For the purpose of this paper, our sample was reduced to those organizations that indicated to be using Linux to at least some degree. A total of 95 cases remained available for further analysis.

\section{Empirical Findings}

In this section, we provide the results of our quantitative analysis. We first report on the type of external support used by the organizations in our sample. We subsequently investigate the influence of a number of factors on the type of external support used by organizations. As can be seen in Fig. 1, we anticipate that 6 factors in two main contexts may have an influence on the type of support used.

\subsection{Types of External Support}

Respondents were asked to indicate which types of external support were used by the organization. We distinguished between three types of external support: (1) vendor support offered by an OSS vendor (e.g., a support contract from RedHat); (2) the services of a third party (e.g., a consultant or service provider); and (3) the OSS community.

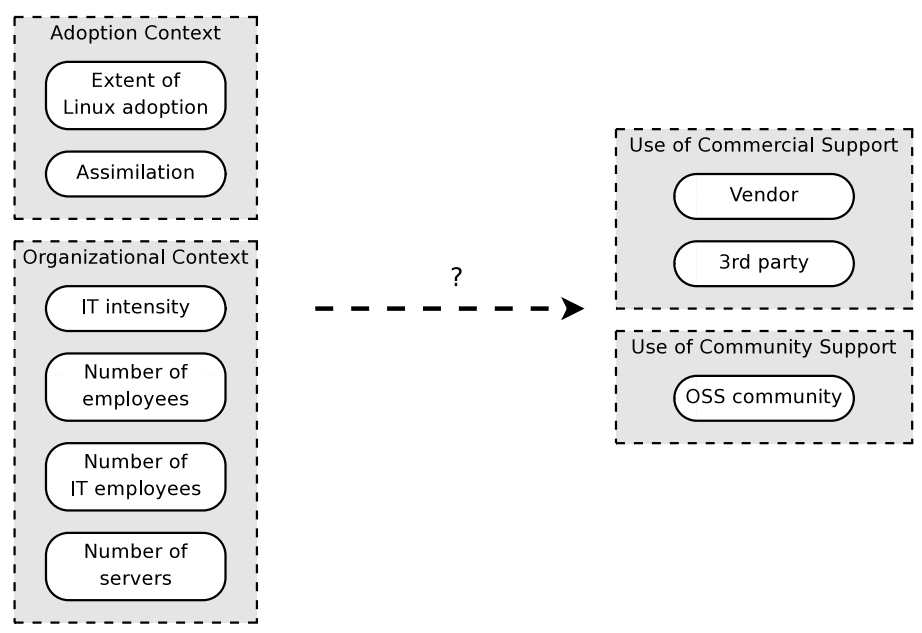

Fig. 1. Factors Potentially Influencing the Use of External Support 

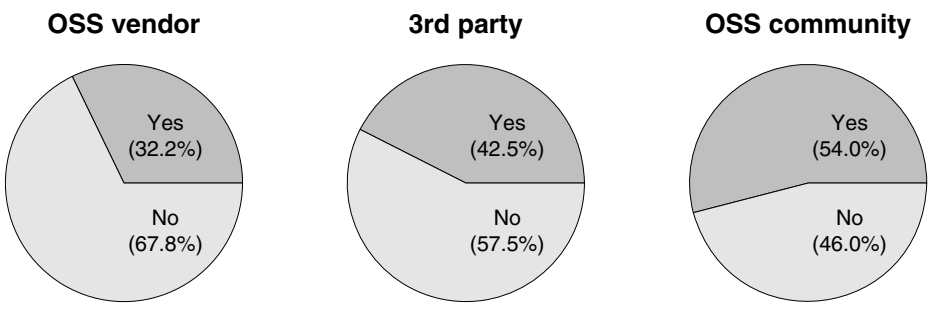

Fig. 2. Use of Various Types of External Support

Table 1. Use of Different Types of External Support

\begin{tabular}{|c|c|c|c|c|}
\hline Vendor & 3rd Party OSS & Community & $\mathrm{N}$ & $\%$ \\
\hline ० & o & o & 15 & $17.2 \%$ \\
\hline$\circ$ & ○ & $\bullet$ & 21 & $24.1 \%$ \\
\hline ○ & $\bullet$ & $\circ$ & 14 & $16.1 \%$ \\
\hline o & • & • & 9 & $10.3 \%$ \\
\hline$\bullet$ & ० & o & 7 & $8.0 \%$ \\
\hline • & o & • & 7 & $8.0 \%$ \\
\hline$\bullet$ & $\bullet$ & ० & 4 & $4.6 \%$ \\
\hline \multirow[t]{2}{*}{$\bullet$} & $\bullet$ & $\bullet$ & 10 & $11.5 \%$ \\
\hline & & Total: & $\overline{87}$ & $100.0 \%$ \\
\hline
\end{tabular}

o: no $\bullet$ : yes

Answers for each type of external support were recorded using a boolean variable, indicating whether the organization used this particular type of support. A summary of the responses is provided in Fig. 2 and Table 1 Fig. 2 shows the proportion of organizations indicating whether they used each type of external support. Given the fact that there are three types of external support, there are $8\left(2^{3}\right)$ possible ways in which the three types of support can be combined. The frequencies at which each combination is used by the organizations in our sample is displayed in Table 1. Cases with missing values have been deleted from this analysis.

The responses show some interesting patterns. First, it is noteworthy that $54.0 \%$ of the organizations indicated to be using the OSS community as a source of support. Moreover, $24.1 \%$ of the organizations relied exclusively on the OSS community for external support. In our qualitative study, it did not appear that the OSS community was such an important source of support [14]. Relying on the OSS community had two main disadvantages: the support was not guaranteed, and searching for a solution to a problem may require more effort. Among the commercial sources of external support, the use of a third party is the most popular choice of organizations 1

Remarkable is that a considerable proportion of organizations $(17.2 \%)$ did not use any type of external support. In addition, $41.4 \%$ of the organizations in our study did not use commercial support. These organizations therefore strongly relied on their internal

\footnotetext{
${ }^{1}$ We use the term "commercial support" to refer to the use of vendor support and/or a third party.
} 
resources to support their OSSS systems. The other $58.6 \%$ of organizations indicated to use commercial support.

\subsection{Relationship with Assimilation Stage}

It is possible that the need for external support evolves as organizations extend their use of OSS. For example, organizations may need external support in the early assimilation stages, since no internal knowledge is available. Over time, the organization may build up the required expertise internally, which makes the use of external support obsolete. We observed the desire to provide support for OSS internally in a number of our case studies [7 14].

Therefore, we performed some analyses to determine whether the type of external support chosen may be influenced by the assimilation stage reached by the organization. We decided to divide the organizations in our sample into two groups, distinguishing between those organizations that made very limited use of OSSS, and those that rely extensively on OSSS. In order to make this distinction, we used the assimilation stage reached by the organization. This information was obtained in the first survey [15]. The Guttman scale developed by Fichman and Kemerer [5] was used to classify organizations into 7 different assimilation stages. Organizations that are situated in stages 0 to 3 have not yet made a formal decision to adopt OSSS. They have progressed at most to the trial or evaluation stage, where the use of OSSS is still being considered. Organizations in the latter stages (4 to 6) have made a formal decision to adopt, and are using OSSS in a production environment. It therefore appears that stage 4 is a logical boundary between assimilation stages. Organizations that have not progressed beyond the trial stage will be termed "non-adopters", while those that have will be termed "adopters"

Given the nominal (binary) nature of our data, a series of $\chi^{2}$-tests were performed to investigate whether the use of the three types of external support were related to the dichotomous non-adopter/adopter distinction. The results are displayed in Table 2 and show a significant effect only with respect to the use of the OSS community. Investigation of the data showed that adopters were far more likely than non-adopters to use the services of the OSS community.

To further investigate the influence of the assimilation stage reached by the organization on the type of support used, we performed three logistic regressions. In each regression, the assimilation stage reached by the organization acted as the independent variable, while the dependent variable was successively a binary variable indicating whether the organization used vendor support, a third party or the OSS community. Results showed that the assimilation stage only had a significant relationship with the use of the OSS community, indicating that organizations that reached a higher assimilation stage were more likely to use the OSS community. Hence, these results are consistent with those in Table 2

In order to provide more insight into the support usage of adopters, we provide a similar overview as in Fig. 2 and Table 1 limiting our sample to adopters. As can be seen from Fig. 3, our data shows that among adopters, $75.4 \%$ of the organizations were using the services of the OSS community. This confirms that the OSS community is

\footnotetext{
${ }^{2}$ Please note that even non-adopters have indicated to be using Linux. However, they are only using Linux in a trial phase.
} 
Table 2. Relationship between Use of External Support and Assimilation Stage

\begin{tabular}{|c|c|c|c|c|}
\hline & \multicolumn{4}{|c|}{ Adopter? } \\
\hline & & No & Yes & Total \\
\hline \multirow[t]{3}{*}{ OSS vendor $(a)$} & No & $23(20.3)$ & $36(38.7)$ & 59 \\
\hline & Yes & $8(10.7)$ & $23(20.3)$ & 31 \\
\hline & Total & & 59 & 90 \\
\hline \multirow[t]{3}{*}{ 3rd party $(b)$} & No & $18(17.4)$ & $32(32.6)$ & 50 \\
\hline & Yes & 14 (14.6) & $28(27.4)$ & 42 \\
\hline & Total & & 60 & 92 \\
\hline \multirow[t]{3}{*}{ OSS community $(c)$} & No & $26(13.6)$ & $14(26.4)$ & 40 \\
\hline & Yes & $4(16.4)$ & 44 (31.6) & 48 \\
\hline & Total & & 58 & 88 \\
\hline
\end{tabular}

Numbers between brackets indicate
the expected count for each cell
$\begin{aligned} & \text { (a) } \chi^{2}=1.563, d f=1, p=.211 \\ & \text { (b) } \chi^{2}=.072, d f=1, p=.789 \\ & \text { (c) } \chi^{2}=31.181, d f=1, p<.001 * * *\end{aligned}$
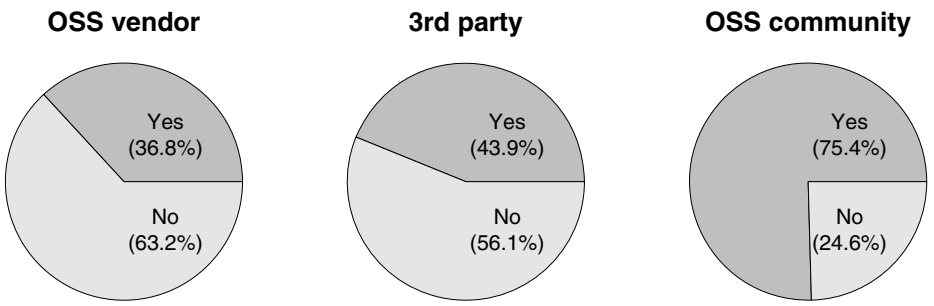

Fig. 3. Use of Various Types of External Support by Adopters

an important source of support to adopters. Table 3 confirms that the proportion of organizations relying on the OSS community is quite high. It further shows that $40.4 \%$ of adopters are not using any type of commercial support, while the other $59.6 \%$ uses at least the services of a third party or a support contract.

\subsection{Relationship with Extent of Linux Adoption}

Similarly, we investigated if the type of external support used was related to the extent of Linux adoption. In the first survey, we determined the extent of Linux adoption measured on a 7-point Likert scale, ranging from "no usage" to "to a very large extent". We performed a series of $t$-tests to investigate whether the degree of Linux adoption was related to the use of any of the three types of external support. The results in Table 4 show that a significant effect only exists with respect to the use of an OSS vendor and indicate that organizations that make use of an OSS vendor exhibit a larger extent of adoption. 
Table 3. Use of Different Types of External Support by Adopters



Table 4. Relationship between Use of External Support and Extent of Linux Adoption

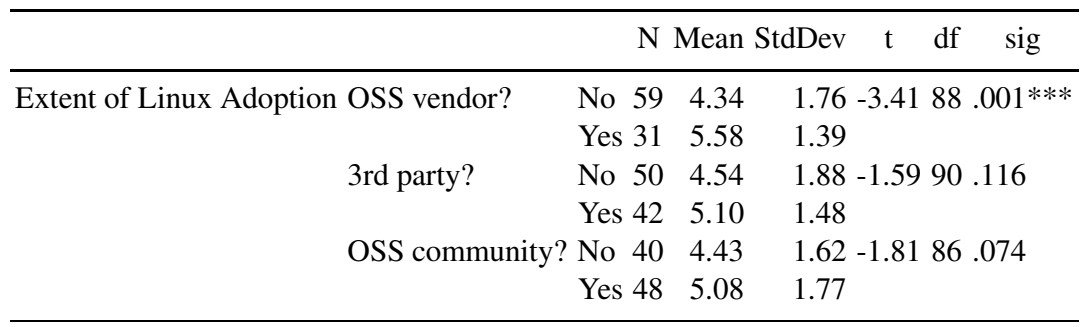

We performed 3 logistic regressions to further explore the impact of the extent of Linux adoption on the type of support used. In each of these regressions, the extent of Linux adoption was entered as the independent variable. The dependent variable in each regression was successively a binary variable indicating whether the organization used vendor support, a third party, or the OSS community. Results showed that the extent of Linux adoption was significantly related to the use of vendor support; organizations with a large extent of Linux adoption are more likely to use vendor support. These results are consistent with our previous analyses in Table 4

\subsection{Relationship with Organizational Characteristics}

Research has shown that organizational characteristics are important in the adoption of technologies [411]. More specifically, the size of the organization can be used as a proxy of the resources available to the organization to adopt and implement new technologies. These resources may also have an impact on the type of external support that is used. If the organization has few resources, it will probably need to outsource part of the support tasks.

Therefore, we investigated if the use of external support is related to characteristics of the organization, such as (1) the number of servers in use by the organization; (2) the number of employees; and (3) the number of employees in the IT department. Since examination of our data showed that these variables were not normally distributed, the 
Table 5. Relationship between Organizational Characteristics and External Support Usage

\begin{tabular}{|c|c|c|c|c|c|c|}
\hline & & $\mathrm{N}$ & Mean & $\mathrm{dDev}$ & $\mathrm{t} \quad \mathrm{df}$ & $\operatorname{sig}$ \\
\hline \multirow[t]{6}{*}{ Number of servers } & \multirow[t]{2}{*}{ OSS vendor? } & No 59 & 2.44 & 1.20 & -0.7587 & .454 \\
\hline & & Yes 30 & 2.66 & 1.42 & & \\
\hline & \multirow[t]{2}{*}{ 3rd party? } & No 50 & 2.72 & 1.14 & 1.8589 & .068 \\
\hline & & Yes 41 & 2.24 & 1.36 & & \\
\hline & \multirow[t]{2}{*}{ OSS community? } & No 40 & 1.97 & 1.10 & -4.2385 & $.000 * * *$ \\
\hline & & Yes 47 & 3.03 & 1.22 & & \\
\hline \multirow[t]{6}{*}{ Number of employees } & \multirow[t]{2}{*}{ OSS vendor? } & No 58 & 5.01 & 1.36 & -1.0687 & .290 \\
\hline & & Yes 31 & 5.33 & 1.36 & & \\
\hline & \multirow[t]{2}{*}{ 3rd party? } & No 49 & 5.15 & 1.36 & 0.4789 & .643 \\
\hline & & Yes 42 & 5.02 & 1.32 & & \\
\hline & \multirow{2}{*}{ OSS community? } & No 39 & 4.67 & 1.33 & -2.7685 & $.007 * *$ \\
\hline & & Yes 48 & 5.45 & 1.29 & & \\
\hline \multirow[t]{6}{*}{ Number of IT employees } & \multirow[t]{2}{*}{ OSS vendor? } & No 59 & 1.28 & 1.17 & -0.7088 & .485 \\
\hline & & Yes 31 & 1.47 & 1.35 & & \\
\hline & \multirow[t]{2}{*}{ 3rd party? } & No 50 & 1.47 & 1.11 & 1.1790 & .246 \\
\hline & & Yes 42 & 1.17 & 1.34 & & \\
\hline & \multirow[t]{2}{*}{ OSS community? } & No 40 & 0.96 & 1.04 & -2.8986 & $.005 * *$ \\
\hline & & Yes 48 & 1.68 & 1.29 & & \\
\hline
\end{tabular}

natural logarithm of these measures was used in our further analysis. Examination of these transformed variables showed that they were normally distributed.

To test these relationships, we performed a series of $t$-tests to determine if the use of any of the three types of external support was related to the three aforementioned organizational characteristics. The results in Table 5 show that a significant effect existed for all three characteristics with respect to the use of the OSS community. Organizations that rely on the OSS community have a large number of servers in use and have a large number of employees, both within the organization and within the IT department.

We further investigated these relationships by performing nine logistic regressions. Each regression investigated the impact of one independent variable (i.e., number of servers, employees and IT employees) on a binary dependent variable (i.e., the use of vendor support, a third party and the OSS community). Results showed that the number of servers, employees and IT employees were significantly related to the use of the OSS community. The larger these numbers, the more likely the organization was using the support of the OSS community. Therefore, these results are consistent with our analysis in Table 5

\subsection{Relationship with IT Intensity}

The previous analyses suggest that the resources of the organization have an influence on the type of external support that is used by the organization. Until now, we used the size of the organization as an indicator for the resources of the organization. However, some organizations make more intensive use of IT compared to other organizations. More IT intensive organizations will have more technical IT resources given the 
Table 6. Relationship between IT Intensity and External Support Usage

\begin{tabular}{|c|c|c|c|c|c|}
\hline & $\mathrm{N}$ & Mean & dDev & $\mathrm{t} \quad \mathrm{df}$ & sig \\
\hline \multirow[t]{2}{*}{ IT intensity OSS vendor? } & No 58 & 0.48 & 0.20 & -0.3386 & .746 \\
\hline & Yes 30 & 0.49 & 0.21 & & \\
\hline \multirow[t]{2}{*}{ 3rd party? } & No 49 & 0.53 & 0.19 & 2.4488 & $.017 *$ \\
\hline & Yes 41 & 0.43 & 0.20 & & \\
\hline \multirow[t]{2}{*}{ OSS community? } & No 39 & 0.41 & 0.20 & -3.6684 & $.000 * * *$ \\
\hline & Yes 47 & 0.56 & 0.18 & & \\
\hline
\end{tabular}

centrality of IT in the organization. We therefore define an "IT intensity" measure as a proxy for the technical resources of the organization. We calculate this IT intensity measure as the ratio between the number of servers and the number of employees in the organization. A higher IT intensity ratio indicates that the organization uses a large number of servers compared to the number of employees. Hence, the larger this ratio, the more the organization relies on IT.

To investigate whether the IT intensity measure is related to the use of the various types of external support, we conducted another series of $t$-tests. The results in Table 6 show two significant relationships. First, it shows that organizations that do not make use of a 3rd party have a higher IT intensity ratio. Second, it shows that organizations that make use of the OSS community have a higher IT intensity ratio.

An additional 3 logistic regressions were performed using the IT intensity measure as the independent variable. The dependent variable was successively a binary measure indicating whether the organization used an OSS vendor, third party or the OSS community. Results showed two significant relationships: (1) organizations with a higher IT intensity ratio are less likely to use a third party; and (2) organizations with a higher IT intensity ratio are more likely to use the OSS community. Therefore, these results are consistent with our previous analyses in Table 6

\section{Discussion}

The aim of this study was to obtain more insight into the importance of external support in the assimilation of OSSS. An overall summary of the significant effects we have found is shown in Fig. 4 Based on our results, we further discuss three main findings of this research.

\subsection{Commercial Support}

First, and as expected, we have determined that commercial support is important to organizations. Our data shows that $58.6 \%$ of the organizations in our sample indicated to be using the services of a third party and/or a support contract from an OSS vendor (see Table 1). It could be expected that commercial support is especially important in the early assimilation stages, since the organization is less likely to possess the necessary internal expertise. Nevertheless, there are several indications that commercial support remains important for organizations that have deployed OSSS to a larger degree. 


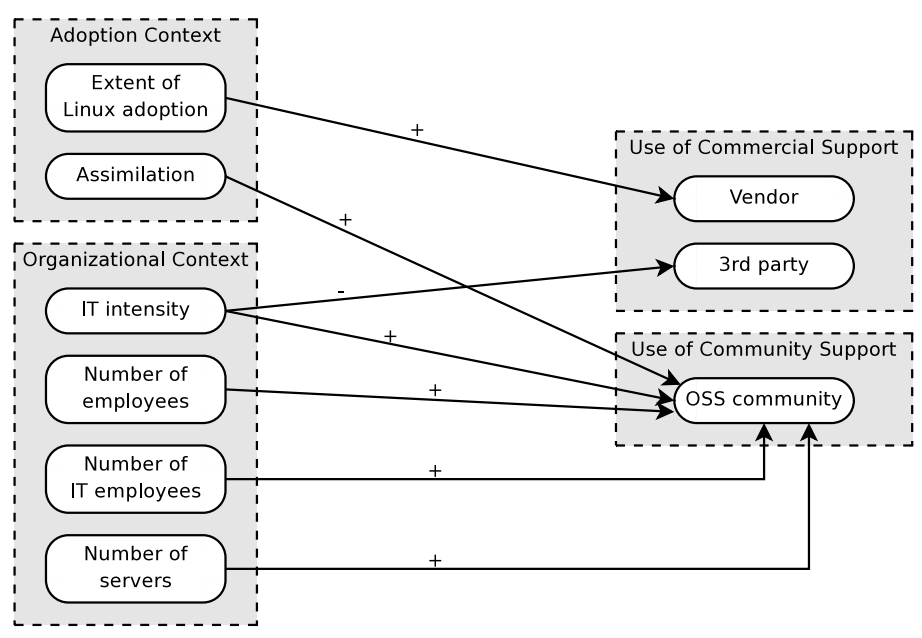

Fig. 4. Summary of Results

A first indication is that when the installed base of Linux increases, organizations are more likely to use a support contract from an OSS vendor (see Table 4). It can be expected that when the Linux installed base increases, the importance of Linux in the organization will also increase. Therefore, organizations will ensure that their missioncritical systems are covered by a support contract. The use of a support contract can also be mandated by audit guidelines [13] or by a vendor that only certifies its application for use with a commercial Linux distribution [16].

A second indication of the importance of commercial support is the fact that commercial support is used by $59.6 \%$ of adopting organizations (see Table 3). The fact that commercial support is important to both organizations in the early and late assimilation stages confirms the findings of our previous quantitative study that did not detect a significant relationship between the availability of external support and the assimilation of OSSS [15].

\subsection{Internal Expertise}

A second important finding of this study is that a considerable proportion of organizations were not using commercial support for OSS. Our data shows that $41.4 \%$ of the organizations in our sample and $40.4 \%$ of adopting organizations (see Table 1 and Table 3 respectively) were not using commercial support. This suggests that these organizations have considerable OSS-related expertise in-house, which makes the use of commercial support obsolete. Although the OSS community may provide a solution to several issues the organization experiences, the IT department will still need to interact with this community and perform the necessary work on their OSS systems. This emphasizes the importance of internal knowledge and expertise on OSS.

One factor that seems to have an impact on the development of internal knowledge on OSS is the centrality of IT in the organization. Organizations that consider IT to be supportive are more likely to use a third party and less likely to rely on the OSS community to support their OSS systems (see Table 6). Since IT does not play a central 
role in these organizations, IT will not be a core competence. Therefore, these organizations may have a rather small IT support staff. As a result, it seems likely that they will prefer-or need - to outsource IT to an external party. If such organizations choose to adopt OSS, it is likely that most operational tasks are outsourced, and thus no internal knowledge on OSS is developed. Conversely, organizations that make more intensive use of IT will be more likely to conduct certain tasks in-house and reduce their dependency on a third party. They will ensure that they have a sufficiently large internal IT staff that is able to maintain the IT infrastructure. It can therefore be expected that IT intensive organizations that adopt OSS will try to support their OSS systems internally. If the organization is new to OSS, sufficient internal knowledge on OSS should be developed. In previous qualitative studies, we have also observed that some organizations will try to develop their knowledge on OSS, in order to be able to provide most or all support internally [7 14].

\subsection{OSS Community}

The most interesting finding of our study is that the support provided by the OSS community is a very important source of support to organizations. Over half of the organizations in our sample $(54.0 \%)$ indicated to rely on the OSS community (see Fig. 2). One may expect that the support of the OSS community is used for resolving smaller issues in the early stages of experimenting with OSSS. The support offered by discussion forums or FAQ lists may assist in resolving many of these smaller issues. However, our data shows that the OSS community is predominantly used by organizations in the late assimilation stages. In fact, $75.4 \%$ of the adopters in our sample have indicated to rely on the OSS community (see Fig. 3). Although we did not question respondents about their perceptions on the support offered by the OSS community, this would suggest that the OSS community is able to provide support of a certain quality.

In addition, we have found that organizations with many resources (in terms of the number of employees, the number of employees in the IT department and the number of servers) and organizations that make more intensive use of IT are more likely to use the OSS community for support. This may indicate that organizations need to overcome some initial barriers and develop some internal knowledge on OSS to make use of this type of support. Employees in the IT department of IT intensive organizations are likely to have a strong technical background. This may also provide them with more knowledge on the OSS phenomenon, which allows them to use the support offered by the OSS community. Organizations that are still new to OSSS may not be familiar with the channels through which support is offered, or may simply not be comfortable in relying on this type of support. Also, they may need to become socialized in the OSS community and familiarize themselves with its norms and behaviour [2], in order to be able to ask for support in a way that is consistent with the expectations of the OSS community. In addition, given the fact that such organizations are new to OSSS, they may encounter certain issues that cannot be efficiently addressed by the OSS community, but require the services of a third party who can provide on-site assistance.

Organizations that rely more intensively on IT and that have adopted OSS are likely to have some mission-critical systems running OSS. It is noteworthy that these organizations make use of the OSS community, since it is conceivable that these organizations 
would prefer not to take any risks and rely on commercial support. However, it is possible that organizations with a strong technical profile are able to obtain support from the OSS community in a more efficient way than from a vendor or consultant. Moreover, it may be that organizations that make advanced use of OSS encounter very specific and complex technical issues to which a local service provider may not know the solution. In those cases, only a few experts may be able to provide a reliable answer, and those experts can be found in the OSS community. Hence, it appears that the OSS community is not only used for resolving relatively small issues, but for answering advanced questions as well. Although we did not obtain data on this, it is likely that service providers will occasionally also resort to the OSS community to resolve issues they encounter with their customers' systems. In that case, the use and value of the OSS community would be even higher than our data currently suggests.

If we consider this phenomenon from the point of view of the organization, it can be said that part of the support tasks are outsourced to the OSS community. In this respect, this can be considered a form of "open sourcing", which is defined as "outsourcing to a global but largely unknown workforce of OSS developers" [1]. Although the term open sourcing has primarily been used with respect to the outsourcing of software development tasks [112], we feel that it can equally well be used concerning the outsourcing of support tasks.

\section{Conclusion}

In this study, we have investigated the role of external support in the adoption of OSS. Our results provide more insight into how the various types of external support are used by organizations. As expected, we have found that commercial support is used by the majority of organizations in our study. It must be noted, however, that commercial support remains important for organizations that have deployed OSS to a large extent. In addition, we established that a considerable proportion of organizations rely on their internal knowledge and, most importantly, that the OSS community is used by the majority of organizations. This indicates that the absence of commercial support should not be an insurmountable barrier to the adoption of OSS. Hence, the value of internal knowledge on OSS and the support provided by the OSS community should not be underestimated. Nevertheless, the OSS community is primarily used by organizations that make intensive use of IT, or that have deployed OSS to a large extent. Hence, primarily organizations with a strong technical background seem to benefit from the knowledge available within the OSS community. This is similar as to how OSS used to be a phenomenon driven by technical enthusiasts, and that was not focused on end users.

Our findings suggest that IT managers should find a well-balanced mix of external support that fulfils the needs of the organization and that is aligned with the knowledge available within the organization. Evidently, this mix of external support may change over time to accommodate changes in the needs and internal knowledge of the organization.

Future research could be performed in a number of ways. First, the survey could be repeated to explore whether the behavior and attitude of Belgian organizations towards external support change over time. Second, it would be interesting to replicate this study in other countries, since differences in the availability of internal and external knowledge on OSS may have an influence on the type of external support chosen. 


\section{References}

1. Ågerfalk, P.J., Fitzgerald, B.: Outsourcing to an unknown workforce: Exploring opensourcing as a global sourcing strategy. MIS Quarterly 32(2), 385-409 (2008)

2. Bergquist, M., Ljungberg, J.: The power of gifts: Organizing social relationships in open source communities. Information Systems Journal 11(4), 305-315 (2001)

3. Dedrick, J., West, J.: Why firms adopt open source platforms: A grounded theory of innovation and standards adoption. In: King, J.L., Lyytinen, K. (eds.) Proceedings of the Workshop on Standard Making: A Critical Research Frontier for Information Systems, Seattle, WA, December 12-14, 2003, pp. 236-257 (2003)

4. Depietro, R., Wiarda, E., Fleischer, M.: The context for change: Organization, technology and environment. In: Tornatzky, L.G., Fleischer, M. (eds.) The Processes of Technological Innovation, pp. 151-175. Lexington Books, Lexington (1990)

5. Fichman, R.G., Kemerer, C.F.: The assimilation of software process innovations: An organizational learning perspective. Management Science 43(10), 1345-1363 (1997)

6. Fitzgerald, B., Kenny, T.: Open source software in the trenches: Lessons from a large scale implementation. In: March, S.T., Massey, A., DeGross, J.I. (eds.) Proceedings of 24th International Conference on Information Systems (ICIS 2003), Seattle, WA, December 14-17, 2003, pp. 316-326. Association for Information Systems, Atlanta (2003)

7. Huysmans, P., Ven, K., Verelst, J.: Reasons for the non-adoption of openoffice.org in a dataintensive public administration. First Monday 13(10) (2008)

8. Li, Y., Tan, C.H., Teo, H.H., Siow, A.: A human capital perspective of organizational intention to adopt open source software. In: Avison, D., Galletta, D., DeGross, J.I. (eds.) Proceeding of the 26th Annual International Conference on Information Systems (ICIS 2005), Las Vegas, NV, December 11-14, 2005, pp. 137-149. Association for Information Systems, Atlanta (2005)

9. Meyer, A.D., Goes, J.B.: Organizational assimilation of innovations: A multilevel contextual analysis. Academy of Management Journal 31(4), 897-923 (1988)

10. Morgan, L., Finnegan, P.: How perceptions of open source software influence adoption: An exploratory study. In: Österle, H., Schelp, J., Winter, R. (eds.) Proceedings of the 15th European Conference on Information Systems (ECIS 2007), St. Gallen, Switzerland, June 7-9, 2007, pp. 973-984. University of St. Gallen, St. Gallen (2007)

11. Rogers, E.M.: Diffusion of Innovations, 5th edn. The Free Press, New York (2003)

12. Shaikh, M., Cornford, T.: Open-sourcing: On the road to the ultimate global source? In: Proceedings of The Second Information Systems Workshop on Global Sourcing: Services, Knowledge and Innovation, Val d'Isère, France, March 10-13 (2008)

13. Ven, K., Van Grembergen, W., Dehaes, S., Verelst, J.: Using COBIT 4.1 to guide the adoption and implementation of open source software. Information Systems Control Journal 3, 31-35 (2008)

14. Ven, K., Verelst, J.: The organizational adoption of open source server software by Belgian organizations. In: Damiani, E., Fitzgerald, B., Scacchi, W., Scotto, M., Succi, G. (eds.) Open Source Systems, IFIP Working Group 2.13 Foundation on Open Source Software, Como, Italy, June 8-10, 2006. IFIP International Federation for Information Processing, vol. 203, pp. 111-122. Springer, Boston (2006)

15. Ven, K., Verelst, J.: The organizational adoption of open source server software: A quantitative study. In: Golden, W., Acton, T., Conboy, K., van der Heijden, H., Tuunainen, V. (eds.) Proceedings of the 16th European Conference on Information Systems (ECIS 2008), Galway, Ireland, June 9-11, 2008, pp. 1430-1441 (2008)

16. Ven, K., Verelst, J., Mannaert, H.: Should you adopt open source software? IEEE Software 25(3), 54-59 (2008) 